\title{
MIDAS: A particle identification tool for the Tragaldabas Cosmic Ray telescope
}

\author{
Y. Fontenla* for the Tragaldabas Collaboration ${ }^{\dagger}$ \\ LabCAF, Instituto Galego de Física de Altas Enerxías, University of Santiago de Compostela, \\ Spain \\ E-mail: yanis.fontenla@rai.usc.es
}

\begin{abstract}
Tragaldabas is a timing-RPC-based Cosmic Ray telescope, a member of the Trasgo family. It is intended for analyzing different aspects of Cosmic Ray Physics. The detector, with a footprint of $1.8 \mathrm{~m}^{2}$, offers tracking capability with an angular resolution of about $0.01 \mathrm{sr}$ and time resolution close to $0.3 \mathrm{~ns}$. This telescope is taking data regularly since April 2015. By means of simulation, a powerful tool for Electron/Muon track identification, MIDAS (Multisampling IDentificAtion Software), has been developed over the Tragaldabas detector. The method is based on a systematic study of several observables that are associated with track reconstruction; such as, the Chi-square of the track-fitting, the number of cells fired over all the planes of the detector or the normalized track length, among others. In the case of electrons, this method is able to give an estimate of its energy. Its accuracy is close to 99\% for Electron-Muon separation using simulated data.
\end{abstract}

36th International Cosmic Ray Conference -ICRC2019-

July 24th - August 1st, 2019

Madison, WI, U.S.A.

\footnotetext{
${ }^{*}$ Speaker.

${ }^{\dagger}$ for collaboration list see PoS(ICRC2019)1177
} 


\section{Introduction}

The study of Cosmic Rays has been one of the most exciting fields in the Modern Physics, and the contribution to the understanding of Nuclear and Particle Physics has been huge.

Cosmic Rays in the Earth surface are detected nowadays by means of different kind of detectors, large area counters and telescopes among them. Recent Resistive Plate Chambers detectors technology (RPCs) allowed to a novel kind of detector systems specially suitable for Cosmic Rays measurements. Thus, the Trasgo family detectors was introduced a few years ago [1].

One of the main features of Trasgo family detectors is their capability of separating muon from electrons using reconstruction algorithms. Moreover, they offer the possibility of making a rough calorimetry of high energy electrons and gammas through the analysis of the shape of the small electromagnetic showers they produce. Tragaldabas [2] is a Trasgo family detector layout, working as a cosmic ray telescope, installed at the Faculty of Physics of the University of Santiago de Compostela, Spain, and taking data since April 2015. It consists on four planes of RPC detectors of $1.8 \mathrm{~m}^{2}$ each, with a distance of $52.5 \mathrm{~cm}$ between planes 1 and 2, 37.9 cm between planes 2 and 3 , and $83.7 \mathrm{~cm}$ between planes 3 and 4, and with a cell granularity of 120 channels per plane.

In this work, we develop and novel algorithm, called MIDAS (Multisampling IDentificAtion Software), intended to identify the different particle species detected by the Tragaldabas system. MIDAS is based in the systematic analysis of the influence that different particles have over some dedicated observables. The behaviour of such observables was parameterized by means of simulation and applied later to real data. Thus, we obtain the electron/muon separation capabilities for Trasgo layouts and for Tragaldabas in particular.

\section{The MIDAS method and algorithm}

The Tragaldabas telescope has been fully simulated within the EnsarRoot simulation and analysis framework [3]. A complete description of the detector geometry was implemented. In addition, the geometry of the building where the detector is located was included as well in the simulation. The Monte Carlo simulation toolkit Geant4 [6] is used in EnsarRoot to propagate the particles through the defined geometries, and the track reconstruction over the detector was performed with the TimTrack method [4]. Figure 1 shows a simulated cosmic ray event over the setup, where a $1 \mathrm{GeV}$ electron interacts in the building materials and produces a secondary shower that can be detected in the Tragaldabas detector. Only the four active RPC planes of the detector are drawn.

The generation of the primary cosmic ray events was done in two different ways: in the simplest case, a dedicated event generator was implemented, where unitary electromagnetic particles (electrons, muons and gamma rays, although gamma rays were not included in the final evaluation) imping in the Tragaldabas system starting randomly from a virtual plane of $200 \times 200 \mathrm{~cm}^{2}$, located $12.7 \mathrm{~cm}$ above the detector and with direction uniformly distributed in $\phi$ and $\cos (\theta)$, with $\theta$ between 0 and $12.8^{\circ}$, and the kinetic energy of the generated particles ranged from 10 to 5620 $\mathrm{MeV}$ for electrons, and from 100 to $5620 \mathrm{MeV}$ for muons; in the realistic case, the CRY Cosmic Ray Event Generator [5] was used to generate primary cosmic rays, which were then propagated over the full setup of building plus detector. 


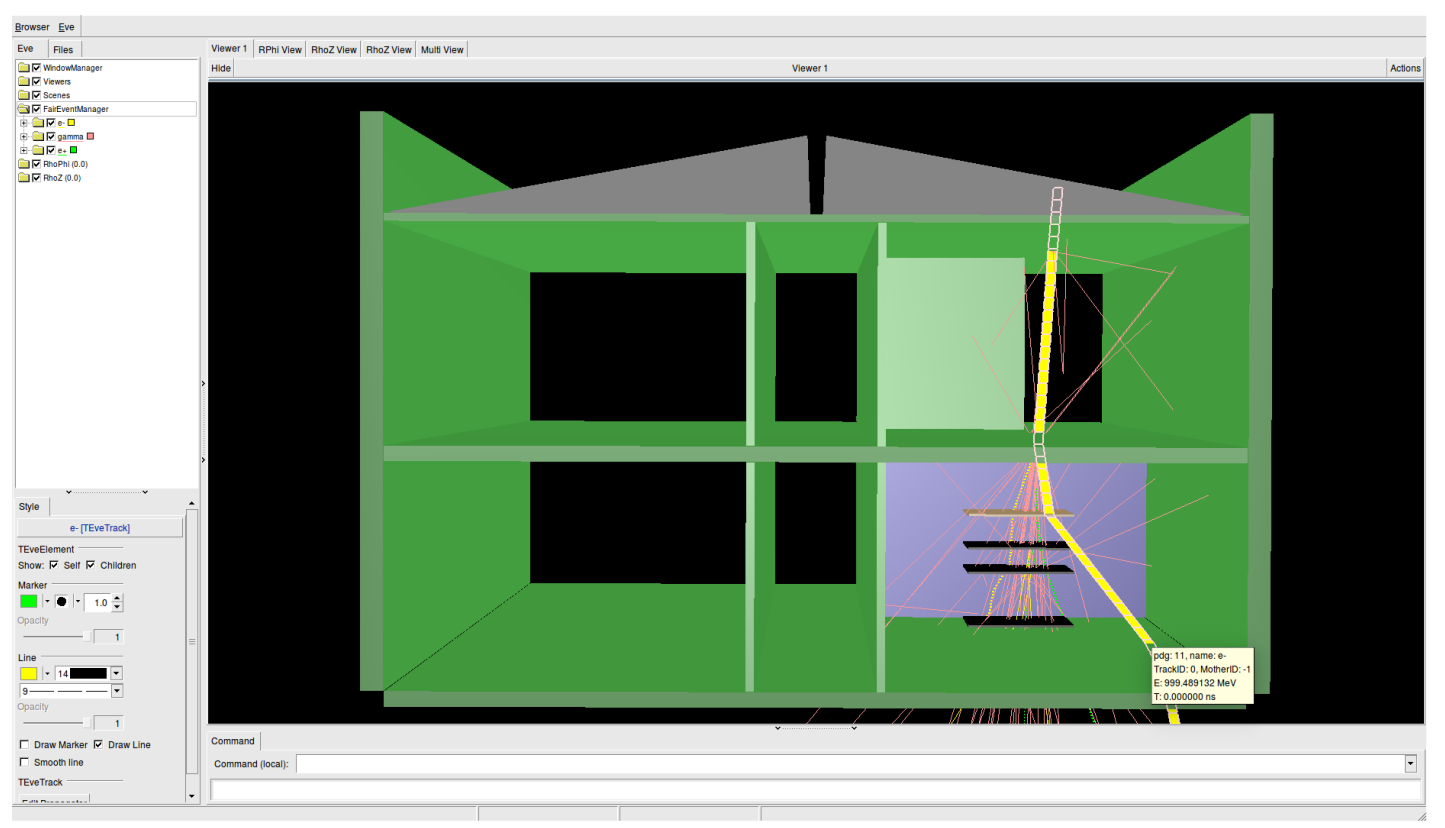

Figure 1: Representation of a simulated cosmic ray event over the full system of building plus detector within the EnsarRoot framework. In the drawn case, a primary electron with an energy close to $1 \mathrm{GeV}$ interacts with the building materials and a secondary shower reaches the Tragaldabas detector. Only the four active RPC planes of the detector are drawn.

For the algorithm development and implementation, the case of the simplest simulation is enough. Thus, some observables were deeply studied for each kind of simulated particle and a phenomenological description was done. Those observables giving the maximum information about the incoming particle nature were found to be: the total multiplicity $\mathbf{M}$, the weighted range $\mathbf{a}_{\mathbf{n}}$ and the chi-square $\chi^{2}$ of the track fitting. They can be defined as follows:

- $\mathbf{M}$ is the total number of hits, that is, valid signals, over the full detector system.

- $\mathbf{a}_{\mathbf{n}}$ is the distance reached by a track or a shower in number of planes, weighted by the hit multiplicity in each plane. It can be described as $a_{n}=\sum_{i}^{p} \mathrm{~m}_{i} \mathrm{n}_{i}$, where $p$ is the plane number, $\mathrm{m}_{i}$ is the multiplicity in each plane and $\mathrm{n}_{i}$ is a weight that goes from 0 (plane 1) to 3 (plane 4).

- $\chi^{2}$ is the chi-square of the track fitting provided by the TimTrack method. Crossing the maximum number of available RPC planes, the value of the $\chi^{2}$ gives an estimation about the deviation of the track from a straight line.

Figure 2 shows the $\chi^{2}$ (left) and $a_{n}$ (right) distributions both muons and electrons. The maximum condition of having a hit in each available plane is requested for a valid tracking. It is observed an important difference between the two kind of particles. Only very high energy electrons (energies higher than $200 \mathrm{MeV}$ ), which are indeed the less in the surface cosmic ray flux, have a similar behaviour to that for muons. But for reconstructed $\chi^{2}$ greater than 10 and $a_{n}$ greater than 7 , one can ensure that an electron is detected with very high probability. It is clearly observed a bump 
around $\chi^{2}=15$ in the electrons distribution. That bump corresponds to the case where an electron is able to travel in straight line through 3 RPC planes but it is strongly deviated in the remaining plane, and its energy is never greater than $100 \mathrm{MeV}$.
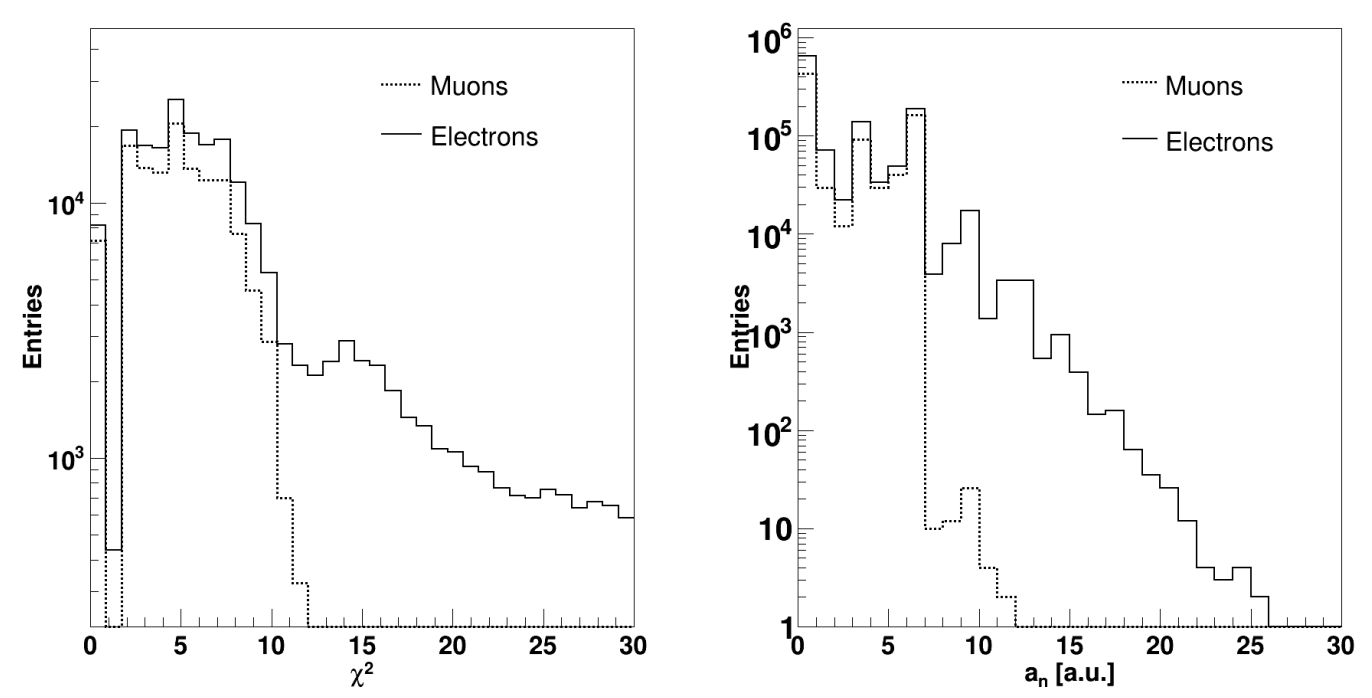

Figure 2: Chi-square distribution for the same amount of generated muons and electrons within all their energy ranges. Only electrons will have a $\chi^{2}$ greater than 12 (Left). $a_{n}$ distributions in the same conditions as for the $\chi^{2}$. In this case, it is clear how a value greater than 7 corresponds to a detected electron (Right).

On the other hand, Figure 3 shows the 2D distributions $a_{n}$ vs $M$ for electrons and muons. Once again, the distinction between distributions is large. Electrons present larger values of multiplicity and $a_{n}$ over the detector, but also the points density is spread in the distribution, while it is concentrated at low $M$ and $a_{n}$ for muons. Indeed, muons have $M=4$ in most of cases, and for all energies, which corresponds to the clean case of the muon crossing the four RPCs planes and giving only, and one only valid signal in each plane. Then, despite the correlation between $M$ and $a_{n}$, the first can be also used for improve the electron/muon separation.

Thus, an appropriate combination of the three proposed observables makes possible the particle identification. The algorithm provides the probability of identification, $\mathrm{P}(\mathrm{Id})$, of being either a muon or an electron. In addition to the particle nature, the minimum energy $\mathrm{E}_{\mathrm{Min}}$ is estimated. In the case when it is not possible to distinguish between different species, the probability of being a certain particle is given by the well known fluxes of cosmic rays at the Earth surface (e.g. table of Kaye\&Laby, NPL).

Starting from a reconstructed single track in the Tragaldabas detector, the flow chart of the MIDAS algorithm when maximum number of active planes are available is presented in Figure 4. As the track reconstruction can be done with different number of RPC active planes, the method also deals with that number and the algorithm design can change slightly.

\section{Results}

The MIDAS algorithm has been implemented in the EnsarRoot framework, and a performance 

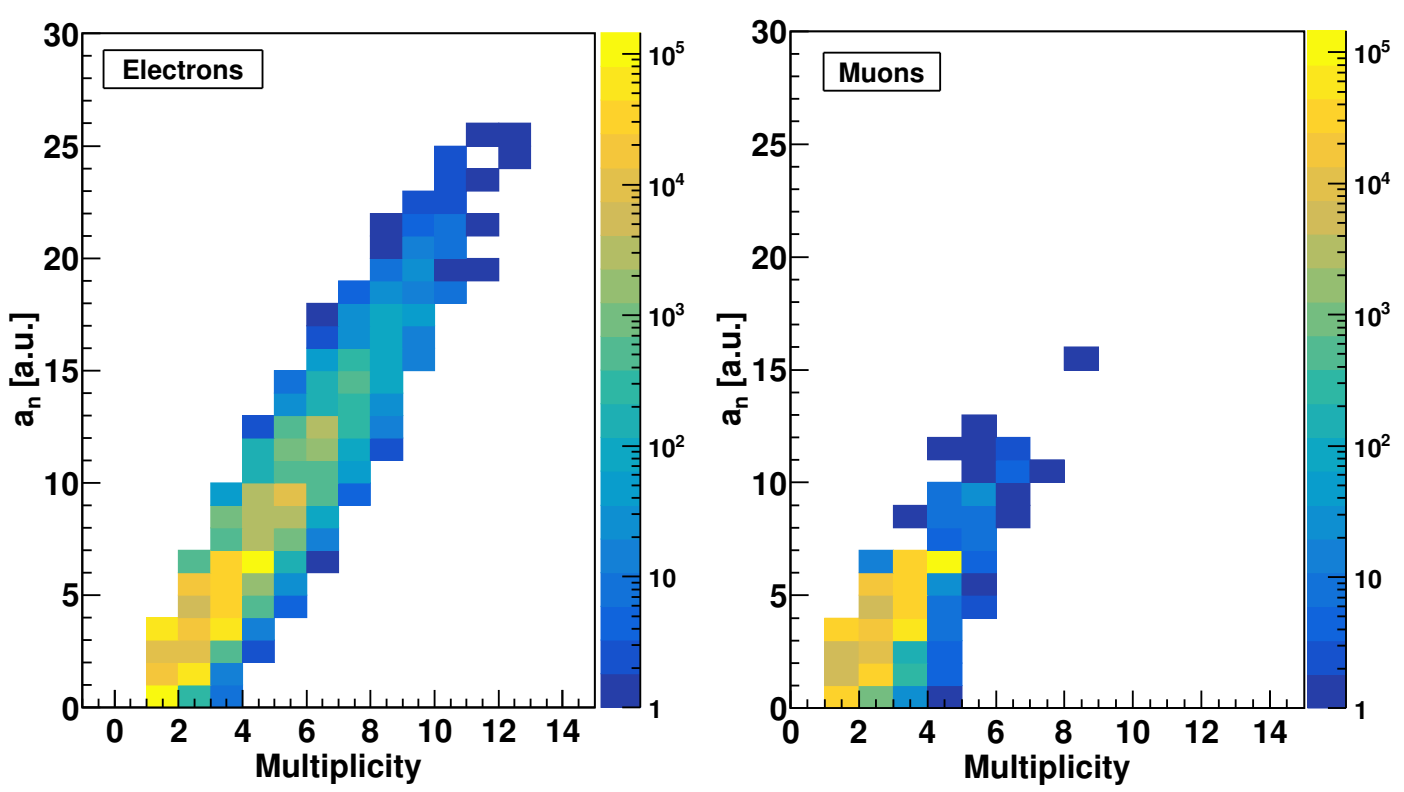

Figure 3: 2D distribution of multiplicity $M$ as a function of the weighted range $a_{n}$, both for electrons (Left) and muons (Right). The distinction between the two kind of particles is clear for $M$ values greater than 4, which corresponds most probably to the clean case of a muon crossing the four RPC planes giving a single hit in each plane.

analysis with simulated data was carried out. The method was tested under both initial event generations described in previous section, and also for the two possible cases of having operative either four RPC planes or only three. As the values of the selected observables change slightly whether your are using four or three detection planes, also the algorithm changes a bit in its design and implementation. In all cases, a trigger condition of having a single track crossing the detector was imposed. In addition, an energy cut was applied to the incoming events so that those particles arriving with not enough energy to pass through $30 \mathrm{~cm}$ of concrete and reach the detector were removed from the input. In the case of having multiple hits in each plane corresponding to a single incoming particle, a minimum $\chi^{2}$ search was applied to select the correct track.

Then, the accuracy of the MIDAS algorithm was estimated. It was calculated a right guess close to $99 \%$ for the case of the simplest simulation, when only different particles impinging the detector are simulated. However, the accuracy decreased down to $90 \%$ for the realistic case of the CRY cosmic ray generator and building included, that is, a misidentification of $10 \%$ occurs. That misidentification was analyzed and two major facts contributes to it. On the hand, in most of the misidentification cases an incoming high energy electron is erroneously identified as a detected muon. This happens in about the $70 \%$ of the times. On the other hand, other incoming particle species like protons or gammas are wrongly identified to be a muon or an electron.

Table 1 summarizes the accuracy probability of the method for the studied cases of realistic or simple simulations, having operative either four or three RPC planes, while Table 2 shows the distribution of the misidentification events in $\%$ for the same analyzed situations. The arrows in the table boxes connecting two different particles mean that an actual incident particle $a$ is misidentified as a particle of type $b$. 


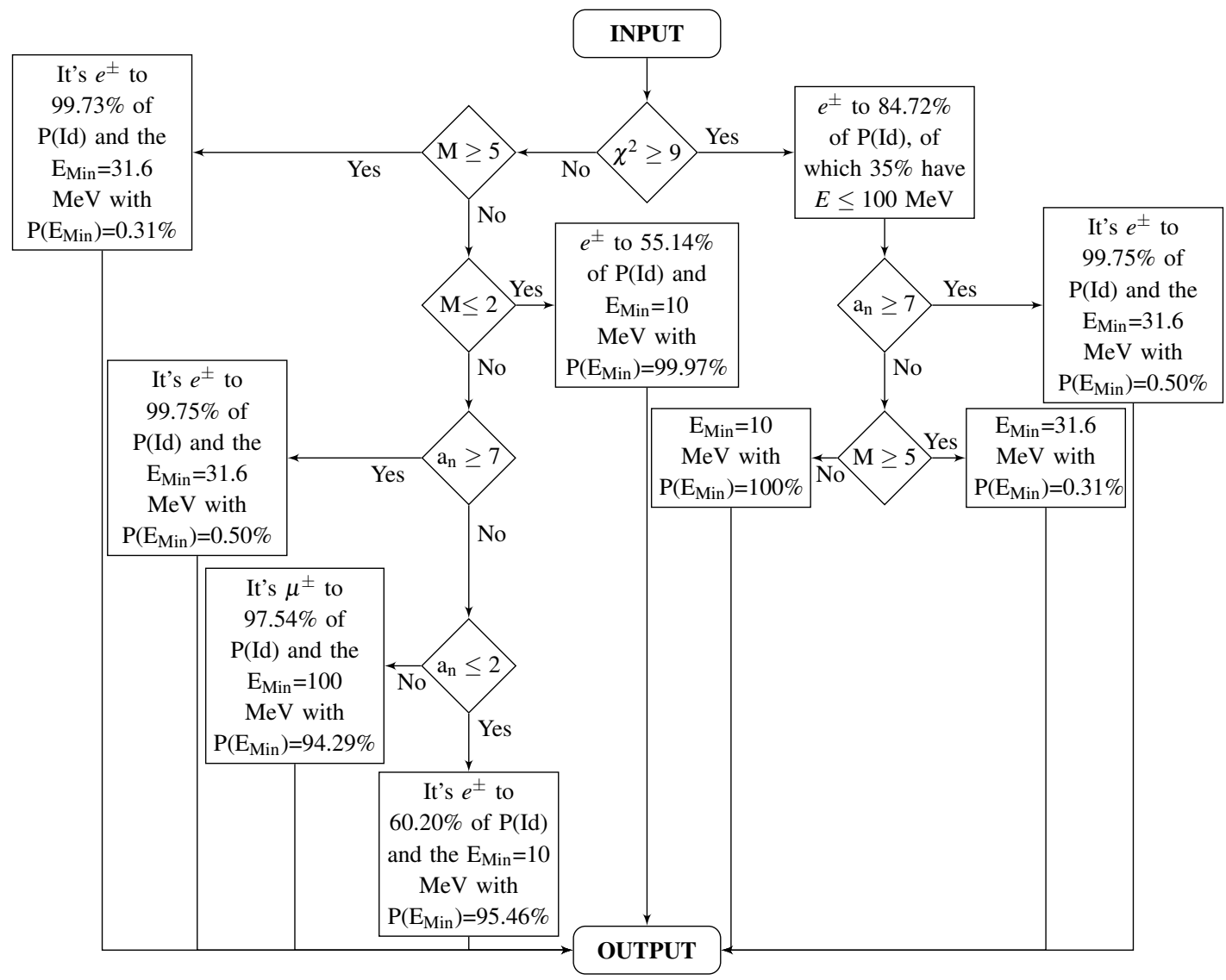

Figure 4: Flow chart for the MIDAS particles identification algorithm in the Tragaldabas detector. The solution procedure when 4 active RPC planes are available is shown. The implementation can change slightly when only 3 or 2 active planes are used.

\begin{tabular}{|c|c|c|}
\hline Active Planes & Realistic Simulation & Simple Simulation \\
\hline 4 & $87.9 \pm 1.5$ & $99.3 \pm 0.2$ \\
3 & $90.2 \pm 1.4$ & $99.3 \pm 0.2$ \\
\hline
\end{tabular}

Table 1: Accuracy results in [\%] for the four analyzed cases. An accuracy close to $100 \%$ is achieved in the simple case, while a value about $90 \%$ is obtained in the realistic cases.

\begin{tabular}{|c|c|c|c|c|c|}
\cline { 2 - 6 } \multicolumn{1}{c|}{} & \multicolumn{5}{c|}{ Misidentification type } \\
\hline Active Planes & $\mathrm{e} \rightarrow \mu$ & $\gamma \rightarrow \mu$ & $\gamma \rightarrow \mathrm{e}$ & $\mathrm{p} \rightarrow \mathrm{e}$ & $\mathrm{p} \rightarrow \mu$ \\
\hline 4 & 68.5 & 0.3 & 20.6 & 9.4 & 1.1 \\
3 & 66.7 & 0.8 & 20.6 & 11 & 0.8 \\
\hline
\end{tabular}

Table 2: Percentage summary of misidentification events. The arrow means that an actual incident particle $a$ is misidentified as a particle of type $b$. It is observed how in most of the cases an incoming electron is wrongly assigned to be a muon. Those correspond to the higher energy electrons.

\section{Conclusions}

The MIDAS particle identification algorithm for cosmic rays has been developed for the Tra- 
galdabas cosmic ray detector. After performing and studying different simulations of cosmic rays, the method uses a combination of different observables to obtain a probability of being a certain particle, and is also able to give a value for the possible energy.

The method was tested in simulated data under different conditions and an accuracy close to $100 \%$ was obtained for the simplest cases, while a value of $90 \%$ was reached in the most realistic simulations. Also, the characteristics of the misidentified events were exposed, being the most frequent case that when an incoming high energy electron is wrongly identified as a muon.

It is expected to have the method working under real data in a short term period.

\section{Acknowledgements}

This work has been financially supported by the European Union Horizon 2020 research and innovation programme under grant agreement No 654002 (ENSAR2) and by the Plan Galego de Investigación, Innovación e Crecemento (I2C) of Xunta de Galicia under project POS-B/2016/015.

Some results presented in this paper were obtained thanks to the access granted to Galicia Supercomputing Center (CESGA) high performance computing resources.

\section{References}

[1] D. Belver et al., TRASGO: A proposal for a timing RPCs based detector for analyzing cosmic ray air showers, Nucl.Inst.Meth. A, 661 (2012) S163-S167.

[2] H. Álvarez-Pol et al., Tragaldabas: A new high resolution detector for the regular study of cosmic rays, J.Phys.:Conf.Ser. 632 (2015), no. 1, 012010.

[3] P. Cabanelas et al., EnsarRoot: The framework for simulation and data analysis for ENSAR, J.Phys.:Conf.Ser. 1024 (2018) 012038.

[4] J.A. Garzón and P. Cabanelas, TimTrack: A matrix formalism for a fast time and track reconstruction with timing detectors, Nucl.Inst.Meth.Phys.Res. A, 661 (2012) S210-S213.

[5] C. Hagmaann et al., Cosmic-Ray Shower Generator (CRY) for Monte Carlo Transport Codes, IEEE Nuclear Science Symposium Conference Record, 2007.

[6] S. Agostinelli et al., GEANT4- A Simulation Toolkit, Nucl. Instrum. Meth. A 506 (2003) 250-303. 